\title{
Levelset and B-Spline Deformable Model Techniques for Image Segmentation: A Pragmatic Comparative Study
}

\author{
Diane Lingrand and Johan Montagnat \\ Rainbow Team, I3S Laboratory UMR 6070 UNSA/CNRS, \\ 930, route des Colles - B.P. 145, \\ F06903 Sophia Antipolis Cedex- France \\ Diane.Lingrand@unice.fr \\ http://www.i3s.unice.fr/ lingrand
}

\begin{abstract}
Deformable contours are now widely used in image segmentation, using different models, criteria and numerical schemes. Some theoretical comparisons between some deformable model methods have already been published [1. Yet, very few experimental comparative studies on real data have been reported. In this paper, we compare a levelset with a B-spline based deformable model approach in order to understand the mechanisms involved in these widely used methods and to compare both evolution and results on various kinds of image segmentation problems. In general, both methods yield similar results. However, specific differences appear when considering particular problems.
\end{abstract}

\section{Motivations}

To model objects and segment images, both explicit 7, 18 and implicit 13, 2 deformable models have been proposed in literature [12. Among these methods, some focus on detecting edges characterized by a high variation of features [10, 3, others on detecting regions characterized by homogeneity of spatially localized properties 17, 11. Some others focus on both approaches.

Implicit deformable models are very commonly represented by levelsets. Levelsets are widely used for 2D image segmentation [14,3,4, and 2D or 3D medical image segmentation [10, 15, 5, 6, 9, 8, among other areas. There are many explicit model representations among which parametric models are the most widely used. In this paper, we consider B-spline parametric models [16] as explicit representation.

Several studies comparing different methods at a theoretical level have been published [1] but without concrete confrontation with real data. Our objective is to propose a comparative study of implicit and explicit deformable model based methods using concrete examples which illustrate the differences between the two approaches. These methods are focused on the determination of a close contour of one or several objects. The initialization consists of a circle or another 


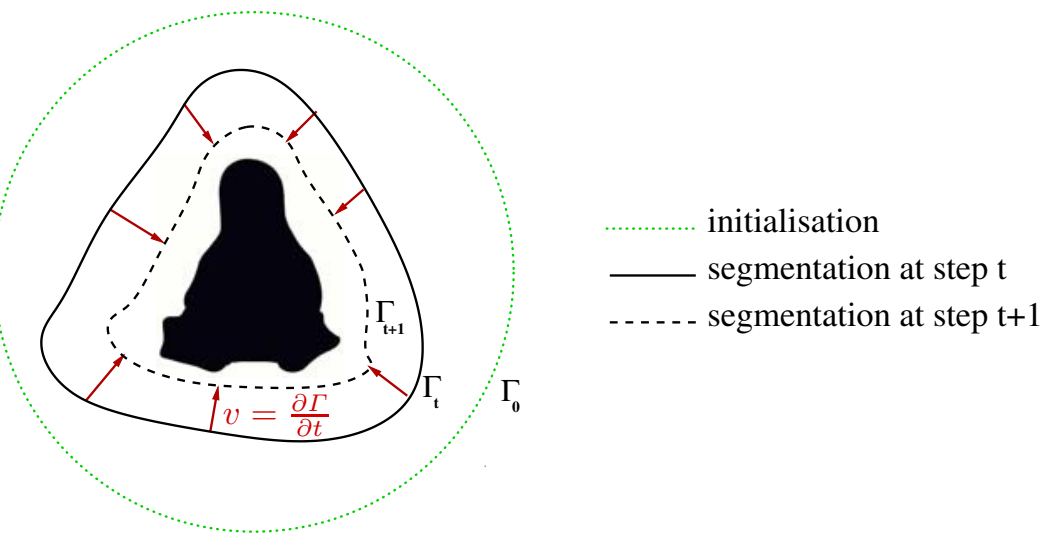

Fig. 1. Principle of image segmentation using deformable curves

closed curve. This curve is iteratively modified according to the law of evolution (see figure 1):

$$
\frac{\partial \Gamma}{\partial t}=F \mathbf{n}
$$

where $\Gamma$ represents the curve, $\mathbf{n}$ represents its normal vector, and $F$ is computed from the image features and the intrinsic curve properties. In practice, we need a representation of the curve, an expression of the force $F$, and a numerical scheme to solve this equation.

\section{Representation of a Curve}

We distinguish three main methods of curve representation: polygonal, parametric and implicit. The polygonal representation is the simplest (see figure 2) but representing a smooth curve implies a model with a large number of points. A parametric model is defined as the set of points $P(t)=(x(t), y(t))$, where $t$ is a real parameter. A lot of papers have been interested by different varieties of splines because of their regularity properties (see figure 2]. Finally, among implicit representations, there is a consensus on the levelset representation which handles model topology changes in a simple and elegant manner.

\section{$2.1 \quad$ B-Splines}

A spline of degree $n$ is defined as a piecewise polynomial of degree $n$. It has an order $(n-1)$ continuity. In order to simplify the parameterization of these splines, uniform B-Splines are considered.

A uniform B-Spline is defined by $n(n \geq 3)$ control points $Q_{i}$ and passes through points $P_{i}$ defined by (see figure 2):

$$
P_{i}=\left(Q_{i-1}+4 Q_{i}+Q_{i+1}\right) / 4
$$



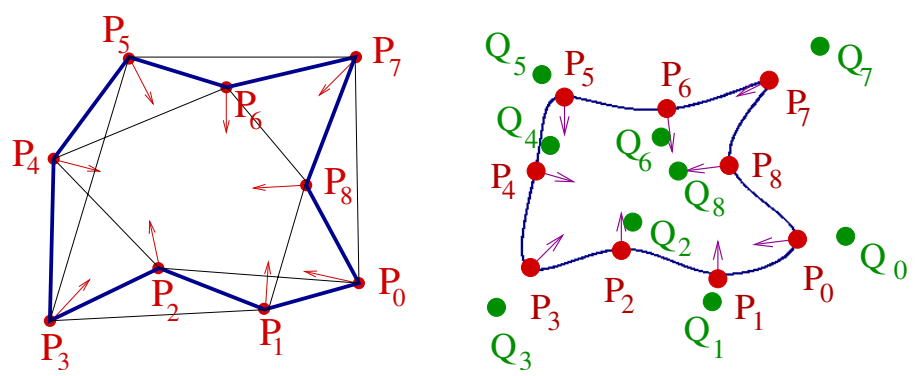

Fig. 2. On the left, the simplest representation of a curve: approximation by a polygon. On the right, uniform B-spline defined by the control points $Q_{i}$ and passing through the points $P_{i}$. Arrows represent normal vectors

Between two points $P_{i}$ and $P_{i+1}$, a curve point $S_{i}(t)$ is defined by the parameter $t \in[0 \quad 1[:$

$$
\begin{aligned}
S_{i}(t)= & {[x(t), y(t)]^{T}=\left(-\frac{1}{6} Q_{i-1}+\frac{1}{2} Q_{i}-\frac{1}{2} Q_{i+1}+\frac{1}{6} Q_{i+2}\right) t^{3}+\left(\frac{1}{2} Q_{i-1}-Q_{i}\right.} \\
& \left.+\frac{1}{2} Q_{i+1}\right) t^{2}+\left(-\frac{1}{2} Q_{i-1}+\frac{1}{2} Q_{i+1}\right) t+\frac{1}{6} Q_{i-1}+\frac{2}{3} Q_{i}+\frac{1}{6} Q_{i+1}
\end{aligned}
$$

We can rewrite this as:

$$
\left\{\begin{array}{l}
x(t)=a_{0}+a_{1} t+a_{2} t^{2}+a_{3} t^{3} \\
y(t)=b_{0}+b_{1} t+b_{2} t^{2}+b_{3} t^{3}
\end{array}\right.
$$

with:

$$
\begin{array}{ll}
{\left[a_{0}, b_{0}\right]^{T}=\left(Q_{i-1}+4 Q_{i}+Q_{i-1}\right) / 6 ;\left[a_{2}, b_{2}\right]^{T}=\left(Q_{i+1}-2 Q_{i}+Q_{i-1}\right) / 2} \\
{\left[a_{1}, b_{1}\right]^{T}=\left(Q_{i+1}-Q_{i-1}\right) / 2 ;} & {\left[a_{3}, b_{3}\right]^{T}=\left(Q_{i+2}-3 Q_{i+1}+3 Q_{i}-Q_{i-1}\right) / 6}
\end{array}
$$

It is then easy to compute the normal vectors and curvature using the first derivative of $x(t)$ and $y(t)$ :

$$
\mathbf{n}=\frac{1}{\sqrt{a_{1}^{2}+b_{1}^{2}}}\left(\begin{array}{c}
-b_{1} \\
a_{1}
\end{array}\right), \kappa=2\left(a_{1} b_{2}-a_{2} b_{1}\right)\left(a_{1}^{2}+b_{1}^{2}\right)^{\frac{3}{2}}
$$

This representation is very light: only a limited number of points $P_{i}$ are needed.

During model evolution, the force $F$ is applied to points $P_{i}$. At each evolution step $t$, the corresponding control points $Q_{i}$ are recomputed by inverting equation (1), in order to determine the B-spline curve (inside and outside regions) and to estimate parameters such as normals and curvature. The computation of normals and curvatures is easy using the above equation. However, during evolution, several parts of the curve may self intersect. It is then necessary to test if this occurs and to split the curve into two parts recursively as needed. Some other tests should be carried out in order to verify the orientation of the curve after splitting operation. If two different curves converge toward the same region (one must be eliminated). However, when two curves intersect, there are different ways of dealing with this depending on the problem to be solved: 
- When a shape prior is introduced, and two objects overlap, one may want to segment both objects and preserve their intersection.

- One may want to obtain distinct but non intersecting regions with a common border.

- One may want to fuse the regions into a single one.

Lastly, the property of uniformity may also be altered by evolution. It is then necessary to rearrange the points along the curve.

\section{$2.2 \quad$ Levelsets}

A $2 \mathrm{D}$ curve is defined by a $2 \mathrm{D}$ distance map which is an image where each pixel has a value corresponding to the distance between this pixel and the curve. This distance is signed assuming, for example, that points inside (resp. outside) the curve have negative (resp. positive) values. The curve is implicitly defined by the isolevel of value 0 .

The normal and curvature are easy to compute on the curve:

$$
\kappa=\operatorname{div}\left(\frac{\nabla u}{|\nabla u|}\right) \text { and } \boldsymbol{n}=\frac{\nabla u}{|\nabla u|}
$$

where $u$ represents the distance map. Normal and curvature can also be computed on a point outside the curve using the isolevel going through this point.

Contrarily to B-splines, the levelset representation implicitly handles problems of topology changes, intersection, superposition or orientation. However, some evolution criterion do not preserve the properties of the distance map: it must be reinitialized regularly.

The levelset representation is simple and easy to implement, but it requires a lot of memory and computations: the whole distance map storage and update is needed for this representation. However, some implementation tricks such as the narrow band may help to reduce the computation time.

\section{Curve Evolution}

A deformable model evolution is steered by an energy minimization process: the solution of the segmentation is defined as the curve minimizing an energy $E$ which depends on the image content (data term) and the curve intrinsic regularity properties (internal term). The data term expresses the characterization of what is searched in an image. By segmentation, we mean the extraction of some objects boundaries from a background. Different characterizations of an object exist: using the boundaries or the region inside the object. Boundaries are characterized by areas of large change of intensity or color. Regions are characterized by constant intensity of color over a uniformed background, range of colors, variance, texture, histogram...

In the following, we will consider the segmentation of a region $\Omega_{\text {in }}$ from a background $\Omega_{\text {out }}$ separated by a curve $\Gamma$ (see figure 3). 


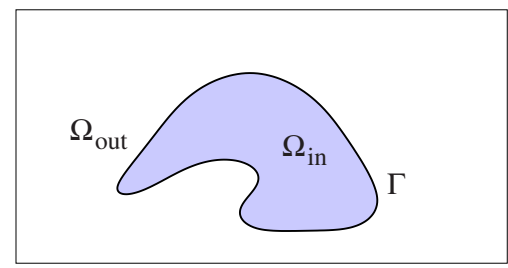

Fig. 3. Notations for the segmentation problem

Segmenting a uniform region from a uniform background. Assuming that we want to segment a uniform region $\Omega_{\text {in }}$ from a uniform background $\Omega_{\text {out }}$, the energy is:

$$
E=\iint_{\Omega_{\mathrm{in}}}\left(I(x, y)-\mu_{\mathrm{in}}\right)^{2} d x d y+\iint_{\Omega_{\mathrm{out}}}\left(I(x, y)-\mu_{\mathrm{out}}\right)^{2} d x d y
$$

where $\mu_{\text {in }}$ (resp. $\mu_{\text {out }}$ ) is the mean intensity of the region $\Omega_{\text {in }}$ (resp. $\Omega_{\text {out }}$ ).

In order to minimize this energy $E$, we derive this expression with respect to the convergence step and obtain the force $F$ :

$$
F=\lambda_{1}\left(I-\mu_{\text {in }}\right)^{2}-\lambda_{2}\left(I-\mu_{\text {out }}\right)^{2}
$$

Many authors have observed that several curves may satisfy the evolution criterion because of the presence of noise in the images and the approximation made in order to model the curves. Among the possible solutions, we can decide to take the curve that minimizes the curve length:

$E=\lambda_{1} \iint_{\Omega_{\text {in }}}\left(I(x, y)-\mu_{\text {in }}\right)^{2} d x d y+\lambda_{2} \iint_{\Omega_{\text {out }}}\left(I(x, y)-\mu_{\text {out }}\right)^{2} d x d y+\lambda_{3} \int_{\Gamma} d s$

Discretized:

$$
E=\lambda_{1} \sum \sum_{\Omega_{\text {in }}}\left(I(i, j)-\mu_{\text {in }}\right)^{2}+\lambda_{2} \sum \sum_{\Omega_{\text {out }}}\left(I(i, j)-\mu_{\text {out }}\right)^{2}+\lambda_{3} L_{\Gamma}
$$

The force is then related to the curvature $\kappa$ :

$$
F=\lambda_{1}\left(I-\mu_{\text {in }}\right)^{2}-\lambda_{2}\left(I-\mu_{\text {out }}\right)^{2}+\lambda_{3} \kappa
$$

\section{Experiments}

Experiments in this section are done on real images taken with a digital camera. We have restricted the study to objects of uniform value on uniform background. Depending on the images, the value is the gray level, or a color component, or some function of a color component. The numeric scheme used in this paper is the simplest: constant iteration. This is not optimal but it is not the subject studied here.

Experiments have been done using a software written in Java by the author. It is available under the GPL License at author web page. 


\subsection{Segmenting Objects of High Curvature}

In this experiment, we want to segment a hand from a uniform background. The image value used is the ratio of the red component from the sum of blue and green component. We use the evolution criterion (2) with both B-splines and levelset representation, each initialized by a circle centered on the image.

Using the B-splines representation, the curve converges easily to one shape but encounter difficulties to segment the fingers (see figure 4). We have helped the segmentation of the fingers by adding points to the model. It is not easy to automatically determine the number of points needed as it depends on the desired precision and curve smoothness.

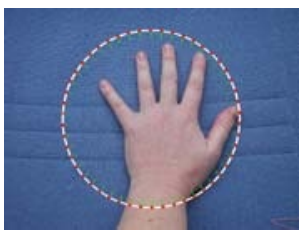

step 5

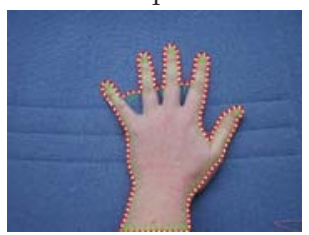

step 45

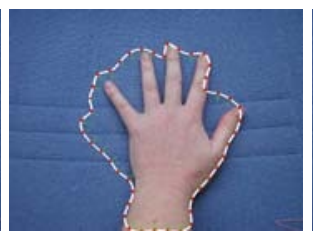

step 15

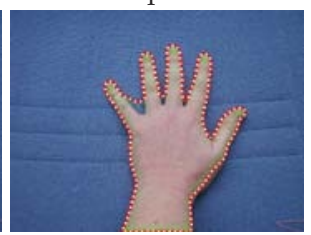

step 55

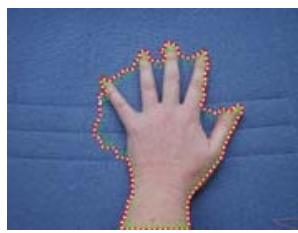

step 25

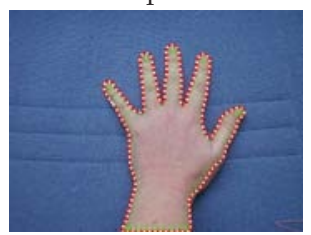

step 65

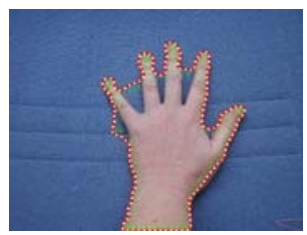

step 35

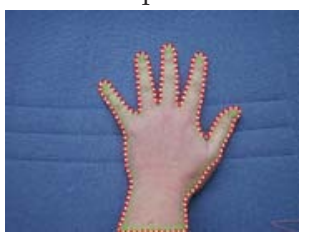

step 75

Fig. 4. B-spline representation. The convergence is reached after 75 iterations. The parameters used are $\lambda_{1}=\lambda_{2}=0.001$ and $\lambda_{3}=1$ for iterations 1 to 15 and $\lambda_{1}=$ $\lambda_{2}=0.0005$ and $\lambda_{3}=1$ for iterations 16 to 60 ) and finally $\lambda_{1}=\lambda_{2}=0.0001$ and $\lambda_{3}=1$. Points have been added in order to be able to represent the curve at iteration 15 (distance between points $P_{i}$ limited from 30 to 50 then from 5 to 15 pixels). We observe that the convergence is difficult between the fingers

Using the levelset method on the same image, we avoid the difficulties of area of high curvature. However, it is necessary to filter the image before the segmentation with a Gaussian filter $(3 \times 3)$ in order to lower the noise level. Figure 5 shows some artifacts that disappear using the filtering (see figure 66).

\subsection{Segmenting Several Regions with Different Colors}

In this experiment, we want to segment the different parts of figure 7 using the mean evolution criterion (2). We use the same initialization as previously: a circle centered on the image. Two problems arise: splitting the curve in several parts and having different regions of different intensities. 


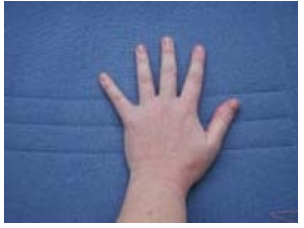

init

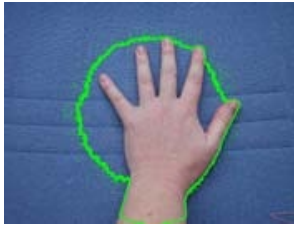

step 9

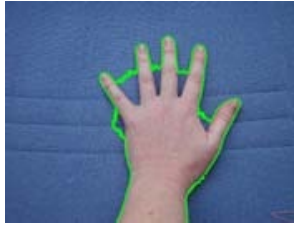

step 19

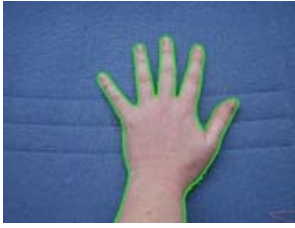

step 29

Fig. 5. Levelset representation. Convergence using 29 iterations with $\lambda_{1}=0.001, \lambda_{2}=$ 0.001 and $\lambda_{3}=1$

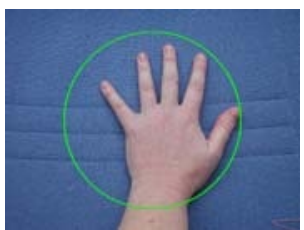

init

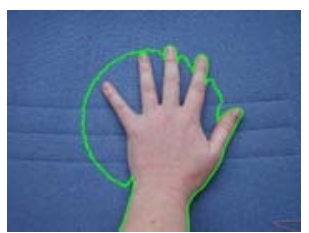

step 5

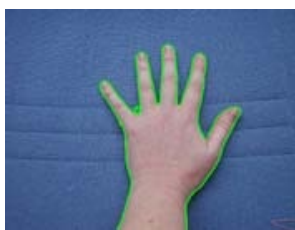

step 10

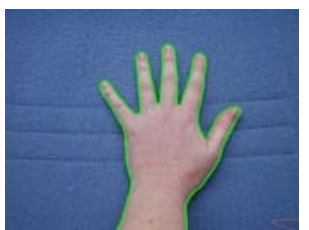

step 11

Fig. 6. Levelset representation with Gaussian filter (8-connectivity). Convergence using 11 iterations with $\lambda_{1}=0.001, \lambda_{2}=0.001$ and $\lambda_{3}=1$

As seen before, the levelset representation intrinsically handles the splitting. However, with the B-spline representation, it is necessary to test when the curve intersect itself and to split it into two curves.

Implementing the evolution criterion (2) using the levelset representation, $\mu_{i n}$ is computed from points of negative distance map value while $\mu_{\text {out }}$ is computed

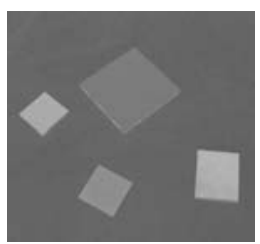

chrom. V comp.

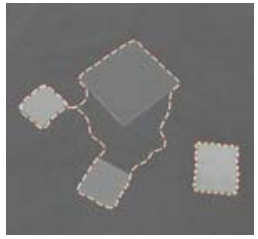

step 115

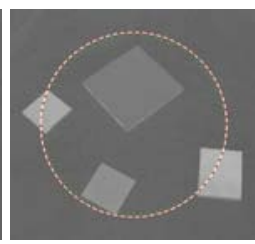

step 1

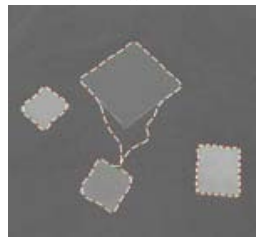

step 297

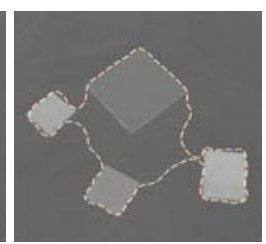

step 54

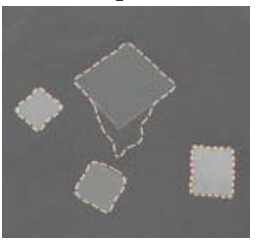

step 298

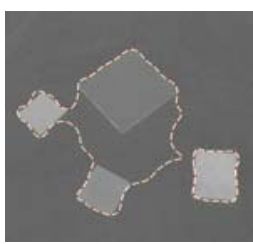

step 55

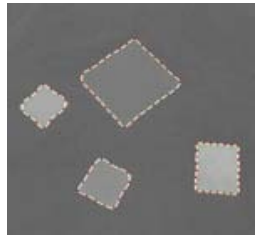

step 520

Fig. 7. Chrominance component $\mathrm{v}$. Using weights $\lambda_{1}=0.01, \lambda_{2}=0.01, \lambda_{3}=1$ and, after iteration number 50: $\lambda_{1}=0.001, \lambda_{2}=0.001, \lambda_{3}=1$. Distance between points $P_{i}$ is limited from 30 to 50 pixels 


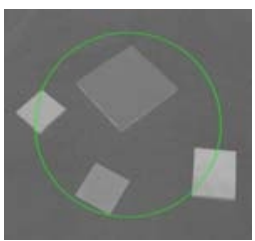

step 1

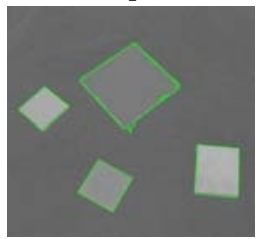

step 87

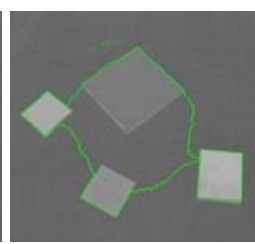

step 66

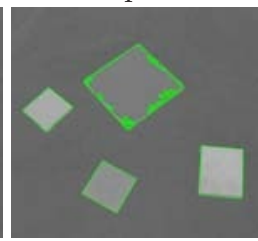

step 99

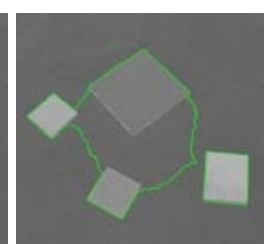

step 68

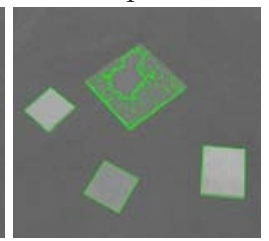

step 129

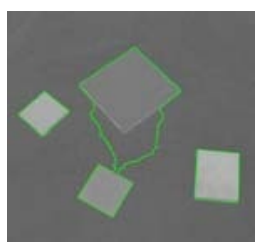

step 80

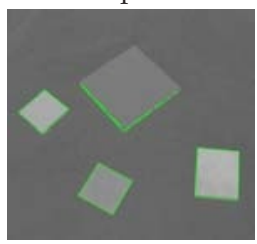

step 139

Fig. 8. Using Gaussian filter $(3 \times 3)$ and weights $\lambda_{1}=0.01, \lambda_{2}=0.01, \lambda_{3}=1$

from points of positive values. It is impossible to distinguish points inside one region from points inside another region: $\mu_{i n}$ is common to all regions. Using the B-spline representation, we need to compute a mask of connected points for $\mu_{i n}$ and $\mu_{\text {out }}$ computation: there is no difficulty to compute a new value $\mu_{i n}$ for each region. Figures 7 and 8 show how the curve is splitted. At the beginning, both seem to converge well. But figure 8 shows that the levelset representation cannot deal with the darker region: the estimated mean is higher and this region has a mean intensity closer to the background. The algorithm does not segment this darker region.

The B-spline representation is more adapted to the segmentation of regions with different intensities. However, in figure 7 the squares are approximately segmented because of the small number of points. It would be necessary to add points to better represent corners. Another solution is to divide the image using the segmentation from the B-spline representation and to refine the result, locally, using levelsets.

\section{Discussion and Conclusion}

Assuming that we want to segment objects of uniform intensity (or value) from a uniform background, both levelset and B-spline representation may fit except that (i) B-spline has difficulties to segment objects with high curvature and (ii) levelset are unable to distinguish one region from the others. However, depending on the class of segmentation problem: high precision on one object, several objects to segment, same or different mean intensity, high curvature, necessity of a light representation, automatic or supervised method, we can make use of both representations.

Considering the problem of several different objects with high precision, we propose to first begin with the B-spline representation. The objects are well separated. Assuming that there is no occlusion in the scene, the image is separated 

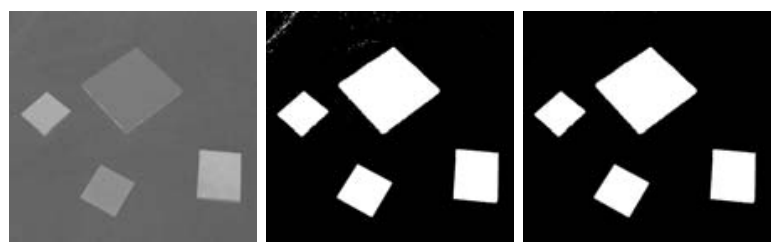

Fig. 9. Original image is first filtered using a Gaussian $3 \times 3$ filter and then thresholded (threshold value $=110$ ). At last, small regions are deleted

in several parts and levelsets are introduced, using the result of B-spline segmentation for initialization. Is it the simplest and fastest method ? As seen in figure 9] there exists an older method that gives a satisfying result: very simple to implement, very fast. After a Gaussian filtering (3x3), the image is thresholded. Objects are well segmented with some small artifacts that are removed considering only regions of large area. This simplicity hide the problem of the choice of the threshold. Considering an application where this threshold can be calibrated (repeated segmentation in the same room with same luminosity ...), this is the method to be chosen. The PDE based methods do not need a threshold determination but are not fully automatic: the weights are important for the convergence.

As a conclusion, we can say that both methods are useful, separately or one helping another. The PDE based methods are more complex but avoid the problem or threshold determination. B-spline can handle different regions but the number of points must be chosen according to the precision needed. Levelset does not need to determine a number of points but cannot manage different regions if their mean intensity are different. Moreover, PDE methods take their importance for more complex problems using more complex regions properties.

\section{References}

1. G. Aubert and L. Blanc-Féraud. Some remarks on the equivalence between 2D and 3D classical snakes and geodesic active contours. IJCV, 34(1):5-17, Sept. 1999.

2. V. Caselles, F. Catte, T. Coll, and F. Dibos. A geometric model for active contours in image processing. In Numerische Mathematik, volume 66, pages 1-33, 1993.

3. V. Caselles, R. Kimmel, and G. Sapiro. Geodesic active contours. International Journal of Computer Vision, 22(1):61-79, 1997.

4. L.D. Cohen and R. Kimmel. Global minimum for active contour models: A minimal path approach. Int. J. of Computer Vision, 24(1):57-78, 1997.

5. M. Droske, B. Meyer, M. Rumpf, and C. Schaller. An adaptive level set method for medical image segmentation. In Proc. of the Annual Symposium on Information Processing in Medical Imaging. Springer, LNCS, 2001.

6. J. Gomes and O. Faugeras. Segmentation of the inner and outer surfaces of the cortex in man and monkey: an approach based on Partial Differential Equations. In Proc. of the 5th Int. Conf. on Functional Mapping of the Human Brain, 1999.

7. M. Kass, A. Witkin, and D. Terzopoulos. SNAKES: Active contour models. International Journal of Computer Vision, 1:321-332, January 1988. 
8. D. Lingrand, A. Charnoz, P.M. Koulibaly, J. Darcourt, and J. Montagnat. Toward accurate segmentation of the LV myocardium and chamber for volumes estimation in gated SPECT sequences. In 8th ECCV, LNCS 3024, pages 267-278. May 2004.

9. R. Malladi and J.A. Sethian. A Real-Time Algorithm for Medical Shape Recovery. In (ICCV), pages 304-310, Bombay, India, January 1998.

10. R. Malladi, J.A. Sethian, and B.C. Vemuri. Shape modeling with front propagation: A level set approach. IEEE Trans. on PAMI, 17(2):158-175, February 1995.

11. T. McInerney and D. Terzopoulos. Deformable models in medical image analysis: a survey. Medical Image Analysis, 1(2):73-91, 1996.

12. J. Montagnat and H. Delingette. A review of deformable surfaces: topology, geometry and deformation. Image and Vision Comput., 19(14):1023-1040, Dec. 2001.

13. S. Osher and J.A. Sethian. Fronts propagating with curvature dependent speed : algorithms based on the Hamilton-Jacobi formulation. Journal of Computational Physics, 79:12-49, 1988.

14. N. Paragios and R. Deriche. Geodesic active contours and level sets for the detection and tracking of moving objects. IEEE Trans. PAMI, 22(3):266-280, 2000.

15. N. Paragios, M. Rousson, and V. Ramesh. Knowledge-based registration and segmentation of the left ventricle: A level set approach. In IEEE Workshop on Applications in Computer Vision, Orlando, Florida, December 2002.

16. F. Precioso and M. Barlaud. B-spline active contour with handling of topological changes for fast video segmentation. EURASIP, vol.2002 (6), pages 555-560, 2002.

17. R. Ronfard. Region-based strategies for active contour models. International Journal of Computer Vision, 13(2):229-251, 1994.

18. D. Terzopoulos, A. Witkin, and M. Kass. Constraints on deformable models: Recovering 3d shape and non rigid motion. Artificial Intelligence, 36(1):91-123, 1988. 